analecta polit. | Vol. 9 | No. 16 | PP. 55-74 | enero-junio | 2019 | ISSN-e: 2390-0067 (en línea) |

Medellín-Colombia

doi: http://dx.doi.org/10.18566/apolit.v9n16.a04

\title{
El sistema político
} como dispositivo de poder en un conflicto medio

The political system as a device of power in an environmental

conflict in Argentina

\section{ambiental en Argentina}
O sistema político como dispositivo de poder num conflito ambiental na Argentina

Fecha de recepción:

\section{ERIKA SACCUCCI}

Doctora en Estudios Sociales de América Latina Universidad Católica de Córdoba

Correo electrónico: erika_sac34@hotmail.com orcid.org/0000-0002-2469-0988 


\section{Resumen}

Este trabajo aborda el conflicto de las y los Vecinos Unidos en Defensa de un Ambiente Seguro (vUdAs) en contra de la permanencia de la empresa Porta Hermanos, productora de bioetanol en Córdoba, Argentina. A partir del análisis de contenido de diez entrevistas en profundidad, ha emergido que uno de los principales dispositivos de poder que se despliegan sobre este conflicto es el sistema político, que funciona como un entramado de poder que controla y limita la lucha. Así, nos hemos propuesto indagar el modo en que se articula este dispositivo y las estrategias que se erigen frente a él. Por último, se ha concluido que la articulación de los mecanismos de poder que produce el dispositivo del sistema político configura estados de excepción que se despliegan sobre los sujetos en lucha a partir de exponerlos al ejercicio del poder arbitrario sin garantías y cercar la lucha política.

Palabras clave: conflicto social, derechos, territorio, Córdoba, análisis de contenido.

\section{Abstract}

This work addresses the conflict of the United Neighbors for the Defense of a Safe Environment (VUDAS) against the permanence of the Porta Hnos company, a producer of bioethanol in Córdoba, Argentina. From the content analysis of ten indepth interviews, it emerges that one of the main devices of power that are deployed on this conflict is the political system, which works as a complex structure of power that controls and limits the struggle. Thus, we have aimed to investigate the way in which this structure is articulated and the strategies that are opposed to it. Finally, it is concluded that the articulation of the power mechanisms that produce the political process device configure states of exception that unfold over the subjects in struggle exposing them to the exercise of arbitrary power without guarantees and encircling their political struggle.

Keywords: Córdoba, social conflict, rights, exceptional state, territory, content analysis.

\section{Resumo}

Este artigo aborda o conflito das e dos vizinhos na Defesa de um Ambiente Seguro (VUDAS) em contra da permanência da empresa Porta Hnos. produtora de bioetanol em Córdoba, Argentina. A partir da análise de conteúdo de dez entrevistas em profundidade, tem emergido que um dos principais dispositivos de poder que se empregam neste conflito é o sistema político, que funciona como uma estrutura de 
poder que controla e limita a luta. Assim, nós nos temos proposto indagar o modo no qual se articula este dispositivo e as estratégias que são erguidas à sua frente. Finalmente, concluiu-se que a articulaçáo dos mecanismos de poder que produzem o dispositivo do sistema político configuram estados de exceção que se desdobram sobre os sujeitos na luta a partir de expô-los ao exercício do poder arbitrário sem garantias e cercar a luta política.

Palavras-chave: Córdoba, conflito social, direitos, território, análise de conteúdo. 


\section{Introducción}

América Latina presenta un patrón de acumulación basado en la concentración de la tierra a partir de procesos de exclusión y desposesión, lo cual da lugar a una multiplicidad de conflictos por el territorio. Esta diversidad de conflictos es producto del avance del capital y la consecuente reconfiguración de las disposiciones de uso y valorización del suelo. Esto ha dado lugar a una mayor presión por la tierra como consecuencia de la reprimarización de las economías (Zibechi, 2006) y de lo que algunos autores han llamado el "consenso de los commodities" (Svampa, 2013). Es en este contexto que se multiplican y profundizan los conflictos por el territorio en América Latina, en general, y en Argentina, en particular.

Así, en la actualidad asistimos a procesos de profundización de las luchas por el territorio, ya sea por su acceso o bien por sus usos y disposiciones, que en muchos casos atentan contra la vida de la población por sus efectos sobre el medio ambiente. O'Connor (2000) plantea la insostenibilidad del modelo actual a partir de lo que llama la "segunda contradicción del capitalismo", la cual refiere a la incompatibilidad que existe entre la feroz avidez de un incremento de ganancias de parte del modelo capitalista y la limitada y cada vez más reducida "capacidad de carga" del medio ambiente.

A partir del desencadenamiento de luchas por el territorio, se despliegan diversos dispositivos de poder para administrar, regular y desactivar el conflicto. Al mismo tiempo, frente al despliegue de los dispositivos de poder los sujetos oponen estrategias que los tensionan. En consecuencia, el conflicto es intrínseco al despliegue de los dispositivos, ya que el ejercicio del poder da lugar a la emergencia de estrategias (Murrillo, 2008).

De allí que en este trabajo interesen particularmente aquellos conflictos por el territorio que se organizan en torno a la lucha por las condiciones ambientales y en defensa de la vida. Particularmente, nos hemos centrado en el conflicto de las y los Vecinos Unidos en Defensa de un Ambiente Seguro (vudas) en contra de la permanencia de la empresa Porta Hermanos, productora de bioetanol en Córdoba $^{1}$. A partir del análisis de contenido, ha emergido que uno de los principales dispositivos de poder que se despliegan sobre este conflicto es el sistema político,

1 El análisis aquí presentado forma parte de la tesis doctoral denominada Los conflictos por la tierra y el ambiente: la (re)producción de los modos de vida, que aborda cinco conflictos por el territorio en Córdoba, donde se realizaron y analizaron 55 entrevistas en profundidad. Lo que aquí se presenta es el abordaje de uno de esos conflictos. 
que funciona como un entramado de poder que controla y limita la lucha. A partir del análisis de las entrevistas, han emergido tres mecanismos de poder: la "oscuridad" del sistema político, la administración de los flujos de información y la arbitrariedad selectiva de la ley. Así, nos hemos propuesto indagar el modo en que se articula este dispositivo y las estrategias que se erigen frente a él en el conflicto vUDAS contra Porta Hermanos. Por último, se ha concluido que la articulación de los mecanismos de poder que producen el dispositivo del proceso político configura estados de excepción que se despliegan sobre los sujetos en lucha a partir de cercar la lucha y exponer los sujetos al ejercicio del poder arbitrario sin garantías.

\section{Dispositivos y estrategias}

Los dispositivos de poder organizan la experiencia del presente, pues es a partir de ellos que se configuran los territorios y sujetos. Regulan las posibilidades de agencia y los límites para la acción, ya que les fijan fronteras a los comportamientos posibles. Su operatoria se despliega como un campo de posibilidades que incitan y/o propician determinadas decisiones y acciones, al tiempo que inhiben y/o prohíben otras. Así, los dispositivos se configuran en la unión de elementos heterogéneos: discursos, instituciones, diseños arquitectónicos, decisiones reglamentarias, leyes, medidas administrativas, enunciados científicos, y proposiciones filosóficas y morales, entre otras, que puedan configurarse y articularse (Foucault, 1994), y están destinadas a la regulación y el control de las acciones de los sujetos y al establecimiento de relaciones sociales reproductivas del orden imperante. A esta red de articulaciones la hemos denominado "dispositivo" y a cada elemento que integra la red "mecanismo". De esta manera, los dispositivos se configuran donde se establece una red de mecanismos y su sentido emerge en virtud de la articulación que establezcan.

Un dispositivo se caracteriza por: 1) definir una serie de conexiones íntimas entre saber y poder; 2 ) establecer la dispersión del poder a través una multiplicidad de dispositivos (la vigilancia, el castigo, el examen); 3) describir la producción de modos de subjetivación del individuo a partir de determinadas técnicas (Abadía, 2003); 4) se trata de un conjunto heterogéneo que incluye diversos elementos: discursos, instituciones, edificios, leyes, medidas policíacas, proposiciones filosóficas; 5) siempre tiene una función concreta inscrita en una relación de poder; y 6 ) resulta del cruzamiento de relaciones de poder y saber (Agamben, 2011).

En definitiva, la doble dinámica de hacer posible y controlar con que operan los dispositivos habilita un juego de acciones y decisiones discrecionales. Así, 
El sistema político como dispositivo de poder en un conflicto medio ambiental en Argentina

pues, los dispositivos son, por un lado, marcos que permiten la creatividad e innovación que requiere la sociedad presente (Guattari \& Rolnik, 2008), y, por el otro, establecen los márgenes de las acciones y decisiones posibles, de modo que las mismas sean manejables y gobernables (Ciuffolini, 2010).

Al mismo tiempo, frente al despliegue de los dispositivos de poder se configuran estrategias que los tensionan. Las estrategias son entendidas como las prácticas desarrolladas por los colectivos en el marco de una disputa agonal que se presenta como un juego de posiciones. Se enfrentan y se oponen directamente al despliegue de los dispositivos de poder y al gobierno de los cuerpos, las poblaciones y los territorios. Esta estrategia designa los procedimientos utilizados en un enfrentamiento con el fin de privar al adversario de sus medios de combate; se trata, entonces, de los medios destinados a obtener la victoria, la elección de soluciones "ganadoras" (Foucault, 1988). En este marco, la lucha es el enfrentamiento directo entre diversos sujetos sociales (De Certeau, 1996), un momento del conflicto donde la tensión deviene en una contienda o disputa. Se trata de la planificación de acciones de manera metódica en virtud de un escenario conflictivo percibido.

\section{Presentación del caso de estudio y decisiones metodológicas}

Porta Hermanos instaló la planta de producción de alcohol en 1995, unas cuatro décadas después de la formación del barrio Parque San Antonio. Sin embargo, en ese momento la regulación de suelo la definía como apta para actividades industriales. Posteriormente, la categorización cambió y desde entonces la planta recibe extensiones en sus permisos de funcionamiento ${ }^{2}$.

El conflicto se inició en $2012^{3}$, cuando las y los vecinos escucharon sonidos de explosión provenientes de la fábrica. Allí se enteraron de que la empresa Porta Hermanos, que hasta el momento producía alcohol y sus derivados, había comenzado a producir bioetanol. Con el pasar del tiempo, los olores nauseabundos

2 Diario La voz, "Aún hay 200 industrias fuera de norma". 8 de noviembre de 2014.

3 Este conflicto se encuentra radicado sobre avenida Valparaíso y la colectora de la Circunvalación, en Barrio San Antonio, y afecta a los vecinos de los barrios Inaudi, San Antonio, Posta de Vargas, Cañitas y 2 de abril. 
y la aparición de múltiples afecciones -como problemas respiratorios, alergias y problemas en la piel- atrajeron la atención de las vecinas, que comenzaron a informarse sobre las consecuencias de este tipo de producción.

La recurrencia de las enfermedades y la aparición de casos de cáncer, púrpura y abortos espontáneos las llevó a contactarse con REDUAs (Red Universitaria de Ambiente y Salud), que realizó un primer relevamiento de las afecciones de la zona. La encuesta detectó que $43,2 \%$ de los vecinos tenía cefaleas persistentes y $34,8 \%$ sufría de conjuntivitis y congestión ocular, por encima de la media parámetro ${ }^{4}$.

A su vez, la articulación con Madres de Barrio Ituzaingó 5 resultó clave para que las vecinas organizadas vincularan la actividad económica con la presencia de las afecciones. El "mapa de la muerte" elaborado por las Madres de Ituzaingó vinculó el uso de químicos con la aparición de las enfermedades y puede hacerse extensivo a la experiencia de vUDAs. De esta manera, las vecinas organizadas en vUDAS reclamaron la relocalización de la planta hacia zonas no habitadas. La demanda asumió mayor relevancia luego de la explosión de la empresa Raponi, ubicada en Alta Córdoba ${ }^{6}$. Esta puso en evidencia la peligrosidad de una planta productora de alcohol en zonas habitadas. Así, el peligro de la permanencia de Porta no solo se vincula a la producción de bioetanol, sino, además, a la magnitud de la posible explosión.

En 2013, los vecinos realizaron una denuncia penal contra la empresa por contaminación, la cual quedó a cargo del fiscal José Mana, que ordenó una pericia científica. Esto resultó de relevancia en tanto era la primera vez que en el fuero

4 Cba 24n. "Acusan que el 50\% del barrio está enfermo por planta de Porta". 13 de agosto de 2013

5 Este caso representa uno de los mayores antecedentes en la lucha medioambiental en Argentina. Este barrio es el más extenso de la periferia de la ciudad y colinda con campos de cultivo. Por esta razón, las fumigaciones eran constantes y ocasionaron muertes, malformaciones en bebés e intoxicación en la población. A partir de las movilizaciones de las madres, se declaró la emergencia sanitaria del barrio y la promulgación de ordenanzas tanto para el barrio como para toda la provincia, que crearon franjas de protección contra las fumigaciones. A su vez, las madres organizadas lograron que en 2012 se realizara un juicio por fumigaciones ilegales, donde fueron declarados culpables un productor sojero y un aeroaplicador. Actualmente se encuentra en curso un nuevo juicio que, a diferencia de aquel del 2012, donde la sentencia fue por mal uso de residuos peligrosos, apunta a la contaminación dolosa, es decir, la relación entre los agrotóxicos y la salud.

6 Raponi era un depósito de productos químicos ubicado en el barrio Alta Córdoba. Esta empresa sufrió una explosión en 2014 y dejó un saldo de una mujer fallecida, 66 personas heridas (varias de ellas de gravedad) una veintena de viviendas destruidas y otro medio centenar con daños. 
El sistema político como dispositivo de poder en un conflicto medio ambiental en Argentina

penal se realizaba una pericia para medir la contaminación en el aire. La pericia oficial se realizó por el Centro de Vinculación de Tecnología Química Industrial (Cetequi), de la Facultad de Ciencias Exactas Físicas y Naturales de la Universidad Nacional de Córdoba, a cargo del ingeniero Yorio, que tiene una cátedra de extensión en articulación con la empresa. Finalmente, los resultados fueron inconcluyentes, ya que "no había pruebas suficientes que pudiesen vincular las afecciones con la empresa", por lo cual la causa fue archivada.

Los primeros años de la lucha se concentraron en acciones directas localizadas en el barrio, al mismo tiempo que se realizaban acciones administrativas en el municipio y la provincia. Sin embargo, al no tener efecto, las vecinas realizaron en 2015 un encadenamiento en el municipio ubicado en el centro de la ciudad. Esta acción es recuperada en la memoria colectiva como un hito que permitió dejar de ser invisibles y el crecimiento del proceso de lucha. A partir del desarrollo del conflicto, el discurso científico ha ocupado una centralidad fundamental. Así, la característica principal de este conflicto ha sido la disputa en torno a los resultados de los estudios que han implicado una discusión de vuDas sobre las formas de medición y los vínculos entre el conocimiento y el poder.

En 2016, las vecinas presentaron un amparo ambiental ante el juez Bustos Fierro, el cual fue rechazado en septiembre de 2017. El amparo era contra la empresa Porta y contra los Ministerios de Ambiente y Energía de la Nación. Ante la apelación de los vecinos, la Cámara Federal ordenó reabrir el caso y le derivó el expediente a Vaca Narvaja, quien ordenó la primera audiencia de conciliación el día 7 de agosto de 2017, a la cual solo concurrieron las vecinas. Es necesario destacar que se trata del segundo caso de amparo ambiental en todo el país.

La selección de estos casos se realizó sobre la base de una compilación de los diversos conflictos articulados en torno al ambiente en Córdoba. Se ponderó la diversidad de los casos y que presentaran elementos novedosos para los estudios medioambientales. El conflicto vuDAs-Porta refiere al único caso en el mundo en que se registra la presencia de una planta productora de bioetanol dentro del ejido urbano, directamente colindante con casas de uso residencial y de gran densidad poblacional. En consecuencia, hemos elegido el "estudio de casos" como estrategia de análisis, donde el caso fue intencionalmente seleccionado en relación con su importancia estratégica, tanto en términos teóricos como empíricos.

De acuerdo con Muñiz (2010), existen diversas perspectivas sobre el uso de los estudios de caso. Para algunos investigadores, este se vincula con un enfoque de investigación: el "caso" constituye el organizador de las diferentes dimensiones de 
análisis que informa y facilita la construcción de las teorías. A su vez, los estudios de caso pueden concebirse como parte de la técnica de recolección de información, el modo de selección de la muestra. Por último, y como aquí proponemos, estos pueden ser abordados como una "estrategia de investigación cualitativa" (Vasilachis de Giardino, 2005). Como señalan Neiman y Quaranta,

[a] diferencia de la grounded theory, [el estudio de caso] puede partir de la utilización de categorías conceptuales para el desarrollo de la investigación y el desarrollo de teoría (Meyer, 2001) y, a diferencia de la etnografía, realiza recortes específicos de la realidad social para su abordaje (Creswell, 1998); a la vez que no se establecen, necesariamente, instancias de reflexividad formalmente formuladas con respecto a la participación del investigador en terreno (2006, p. 216).

Para el estudio de este conflicto se realizaron diez entrevistas en profundidad mediante la implementación de la técnica de la bola de nieve. El análisis de los datos se realizó recuperando herramientas del análisis de contenido. Ello implicó el abordaje de los datos a partir de la emergencia de categorías "en vivo", entrelazándolas y tensionándolas con la literatura que acompaña a nuestro objeto de estudio. Como resultado de ello, obtuvimos una clave de interpretación de esos datos que involucra múltiples discursos: el teórico, el de los actores y el nuestro.

Diseñamos una estrategia de aproximación que consta de varios momentos. En un primer momento se procedió al análisis de contenido de los datos generados durante el trabajo de campo, para luego comenzar a indizar algunos elementos discursivos que podrían advertir de regularidades y relaciones. Este proceso condujo, muchas veces, a encontrar discursos que excedían, en todo o en parte, los marcos conceptuales, lo que exigió un ejercicio analítico de producción de categorías para fundar empíricamente las interpretaciones que hemos ido elaborando. En otras situaciones esos marcos permearon nuestro abordaje y tensionaron nuestra interpretación, al operar recortes específicos sobre los datos, denunciar ausencias y exigir la reapertura del trabajo de campo (Avalle, 2014).

La secuencialidad con que aquí se presenta el método es solo expositiva, pues este implicó un permanente desplazamiento entre el dato y la conceptualización construida a partir de él. Dicha operatoria fue posible gracias a la utilización de un complejo de herramientas articulado en torno a nuestros objetivos de análisis.

En síntesis, recurrimos a la asistencia de la técnica de análisis de contenido que implica la lectura general de toda la documentación y la posterior segmentación e indización de aquellos extractos sugerentes. Luego, como el mismo procedimien- 
El sistema político como dispositivo de poder en un conflicto medio ambiental en Argentina

to de análisis que esta técnica requiere, se procedió a releer estas selecciones para identificar los aspectos característicos que denotaban.

\section{El sistema político como dispositivo de poder}

Las luchas se despliegan en contextos políticos específicos que establecen diversos mecanismos para posibilitar su administración y desactivación. Así, en el análisis de las entrevistas del conflicto vUDAs emergen múltiples alusiones a un "sistema político" difuso y poco claro que se encuentra asociado a los partidos políticos y al Gobierno en todos sus niveles y poderes. Desde estos discursos, el poder político aparece como un dispositivo que se encuentra vinculado al dinero, la coerción y a una profunda vinculación entre los dirigentes políticos y el capital en sus diversas fracciones.

En el discurso de las entrevistadas, el sistema político funciona como dispositivo en tanto emerge en el marco de los conflictos para disciplinarlos, controlarlos y desactivarlos. No se trata de la totalidad del sistema político, en general, sino de la particularidad que asume en los conflictos para su distensión. Su funcionamiento "oscuro" pone al cuerpo en un marco de subyugación y exposición:

EO1: Plata, plata y poder, nosotros creemos que Porta le paga la campańa a Mestre (intendente) y que bueno, que el papá de Porta fue Ministro de Industria que tiene muchos contactos a niveles político, económico, empresarial y yo creo que después de lo que pasó en la municipalidad con el encadenamiento, con la intervención de la policía, el comisario Gómez, que se llegaron distintas organizaciones, creo que quedó plasmado que también la provincia está metida. Porque nosotros todavía no sabemos quiénes dieron las órdenes. Al día de hoy todavía no sabemos quiénes dieron las órdenes ni para que intervenga el comisario Gómez con toda la infantería, ni para (-) porque hay dos instancias, una viene dada por la Fiscalía que decía que el fiscal Mana había mandado a desalojar a ellas, la municipalidad dice que, otra que la municipalidad le había pedido al fiscal Mana que mandara a desalojar, otra que fue el comisario Gómez que fue a desalojar. Nunca se desalojó, la orden creemos que nunca estuvo, el comisario Gómez se manejó con órdenes, hasta ahora no sabemos de quién, al principio parecían que eran de la municipalidad, después parecía que eran del fiscal Mana, al día de hoy todavía no sabemos. Qué es lo que vamos a hacer ahora, pedir un informe para saber quiénes dieron las 
órdenes estas, pero es como todo muy oscuro, es todo muy oscuro, pero para mí, es evidente que la provincia también está metida en esto. ER1: ¿ O sea que no hay registro de una orden en la Fiscalía de envío de infantería? EO1: No hay incluso cuando a ellas no las dejaban pasar la comida... que viene un representante de la nación de Derechos Humanos, cuando lo acompañamos para que intervenga, para ver si nos dejaban pasar la comida, a él. Primero que no lo atendieron, segundo le dijeron que teníamos que llamar a la Fiscalía para pedir la autorización. Cuando él llama a la fiscalía del fiscal Mana, él le dice "No, ya está dada la orden de que se les deje ingresar la comida, de que estas mujeres no tienen (-) no están violando ningún derecho y que se les deje ingresar la comida". Llegamos con esa orden y tampoco nos recibieron [...]. A todo esto, el día viernes nosotros habíamos ingresado los remedios y a ellas nunca les dieron los remedios, todas esas horas que ellas estuvieron incomunicadas nadie se hace cargo (vUDAs, 51:157).

En este extracto emerge un discurso que señala la "oscuridad" del sistema político. Oscuridad en tanto la división de reparticiones y funciones no se respeta en aquellos casos donde interviene el "poder". Se reiteran léxicos que aluden a un "no saber" y a "quién", que expresan la incertidumbre en torno al procedimiento legal.

El sistema de garantías se ve suspendido frente a una acción directa disruptiva -como aquella relatada en el extracto- y la transparencia de los mecanismos legales da paso a la oscuridad que impide conocer el origen de las decisiones. Así, los cuerpos en lucha son destinatarios de una serie de acciones y ejecuciones en las que no es posible discernir su origen. De esta manera, los cuerpos son violentados, pero no les es posible identificar un oponente. Se trata del cuerpo que se enfrenta a un dispositivo de poder despersonalizado, y que (re)produce la exposición y precariedad.

Este funcionamiento entra en contradicción con el sistema de derecho que regula las funciones y los alcances de cada órgano del sistema político y recepta los derechos básicos de los individuos. Este tipo de suspensiones del derecho son propias de los estados de excepción (Agamben, 2004, 2010). Se trata de una "excepción” que se convierte en regla. El cuerpo es desposeído de todo tipo de derecho fundamental y la posibilidad de acceder a remedios o incluso a comida durante una acción directa se encuentra regulada en el marco de un estado de excepción.

7 Los códigos que se encuentran al final de los extractos de entrevista son identificatorios. El primer número refiere a la entrevista mientras que el segundo da cuentas del extracto. Ejemplo: (7: 1), entrevista 7, cita 1. El signo (-) refiere a interrupciones en la enunciación por parte de la/ el entrevistado, mientras que el signo [...] da cuenta de palabras inaudibles en la grabación. 
Este estado de excepción se presenta como un complejo y opaco entramado de relaciones de poder que se despliegan para controlar el conflicto. Se construye una asociación poder-dinero-oscuridad-complicidad donde el sistema político funciona de acuerdo con los intereses del capital, lo que da lugar al ejercicio del estado de excepción. Desde el discurso de los entrevistados, la excepción abarca todos los niveles administrativos, desde los más operativos y barriales hasta aquellos más técnicos. Así, la oscuridad de las complicidades funciona como mecanismo que obstruye que la verdad salga "a la luz".

El término "estado de excepción" resulta útil para caracterizar la sustitución progresiva de la política por formas de control social o violencia física. Refiere a un poder que es ejercido directamente sobre el cuerpo, que busca "reencauzarlo", disciplinarlo y, con él, hacerlo con el territorio.

No existe un estado de excepción, sino estados de excepción, es decir, parcelas de poder que, legal o ilegalmente, escapan de los límites establecidos por el Estado de derecho (Valim, 2018). De este modo, Estado de derecho y estado de excepción no son términos opuestos o contradictorios, sino que deben ser entendidos en estrecha vinculación.

Así, en el discurso de las y los entrevistados, el dispositivo del sistema político produce un estado de excepción sobre los cuerpos que tiene por objetivo desactivar el conflicto. El dispositivo articula los partidos políticos, la justicia, los medios de comunicación y los representantes del Gobierno. Todos ellos se encuentran supeditados a intereses que los trascienden y se vinculan con las necesidades del capital:

En las últimas (pericias ambientales) salen los resultados de altísimos porcentajes de tolueno, cilue(-) cileno, acetaldehído, formaldehído, que es impugnado y que nosotros hemos vuelto a apelar a esto porque bueno, consideramos que nuestras pericias están bien hechas y que es Porta con su poder el que está metiendo la mano en todo esto y no permite que se conozca y que todo esto salga a la luz. Por esto es que tampoco los medios de comunicación nos estaban acompañando, los distintos partidos políticos tampoco nos estaban acompañando, ahora se están sumando o vienen a escuchar pero por una cuestión más electoral, que nosotros también eso lo sabemos, incluso el mismo intendente Mestre, ahora bueno, nos dio la reunión después de que ellas estuvieron encadenadas, después de toda una semana de protestas en la municipalidad y también sabemos que es por el tema de las elecciones (vUDAs, 51:3). 
El poder del oponente es percibido desde los bloqueos que genera, desde el sentimiento de soledad en la lucha cotidiana y desde la falta de apoyos. Desde la imposibilidad de contar con aliados políticos que generen un "contrapeso". Solo es posible torcer esta situación al optar por medidas de acción directa disruptivas. Aquí, la disrupción de la acción se encuentra relacionada con el contexto de elecciones y con la decisión de "trasladar" la acción directa a un nuevo territorio: la municipalidad. El contexto de elecciones genera un escenario político que habilita juegos de poder: realizar una acción directa en la sede de la municipalidad crea un costo político para el intendente que lo podría obligar a oír los reclamos de la organización o, al menos, iniciar un espacio de diálogo. A su vez, esta acción cuenta con un peso simbólico singular en tanto señala directamente a los responsables políticos de la permanencia de la empresa, al tiempo que implica una localización más céntrica. Esto redunda en una mayor visibilidad pública que obliga a las autoridades a responder al reclamo.

Sin embargo, a pesar de la estrategia del colectivo, el Estado de excepción desplegado sobre el conflicto produce que el poder del adversario aparezca como omnipotente y omnipresente, ya que interfiere y busca bloquear toda posibilidad organizativa, inclusive aquella que supone el uso de un espacio privado. El control sobre los procesos organizativos se manifiesta también como control de los flujos de información. Este funciona como mecanismo de poder del dispositivo del sistema político y configura un sistema de vigilancia que se despliega sobre cuerpos singulares. Se trata de un mecanismo individualizante de una porción de la población en lucha:

ER1: ¿¿Han tenido algún contacto con el sr. Porta? EO1: Ellas han tenido contacto en los principios [sic], ahora no; hace un tiempo. Sin tener en cuenta que nosotros tenemos casi todos los teléfonos intervenidos en nuestras casas. Muy poco hablamos por teléfono nosotros. EO2: Ahora hay más ruido, más ruido que antes. ER1: ¿Ustedes se dan cuenta por los ruidos que hacen? EO1: Y porque más de una vez llaman y cortan, llaman y corta, llaman y cortan. A mí eso me pasa todo el tiempo de que llaman y cortan, llaman y cortan o que hablas y no se escucha nada, zzzzzzz. EO2: O una radio. "¿Tenés la radio prendida?". "No, no tengo nada". EO1: Hay como una interferencia. EO2: Aparte ellos siempre se enteran de todo lo que vamos a hacer. EO1: De antemano. EO2: Siempre, por eso lo de la municipalidad fue organizado sumamente en secreto, callado, por un grupo muy chico, cuando ya habíamos decidido todo se les avisó a las organizaciones para que fueran a apoyar, cosa de que no nos... jodieran (risas) y las próximas medidas también las haremos igual, así de callados, organizandonos entre nosotros, cosa de que no se enteren, porque cuando ellos se enteran ya intervienen (vUDAS, 51:25). 
La intervención de los teléfonos permite obtener información "de antemano" y dificulta la efectividad de las acciones directas en tanto se pierde el "efecto sorpresa”. En un juego de posiciones, perder el efecto sorpresa implica la obsolescencia de la acción. Por esta razón, los colectivos optan por desarrollar otros canales comunicacionales y reducir el grupo destinatario de la información como una forma de preservar la efectividad de la acción directa.

Se presenta nuevamente la omnipresencia del adversario que ya había emergido en extractos anteriores. Esta refiere al poder de estar presente en todas partes en situaciones de conflicto:

Cada vez que vamos a hacer alguna actividad así directa, ellos están primero, está la infantería, la policía, han cerrado los portones, ahora fuimos el... el fin de semana anterior estuvimos en la plaza de... Barrio 2 de Abril... Bueno, nos ha costado comunicarnos con otros barrios, entonces en la búsqueda de eso, de compartir con los barrios cercanos, con los que no habíamos estado compartiendo y con los que tuvimos un enfrentamiento en la última toma que hicimos, esto de que nos paramos delante de los camiones hubo conflicto entre los vecinos, entonces decidimos ir a tomar mates en la plaza con ellos, a contarles y demás. Cuando llegamos ya estaba la municipalidad ahí y una ong que se llama Yo Amo Mi Plaza, hablando con los vecinos, antes que nosotros llegáramos. Esas cosas denotan que saben todo lo que hacemos, no sé cómo hacen (vUDAs, 55: 6).

La omnipresencia refiere a la imposibilidad de desarrollar las estrategias de lucha libremente. Lo que se encuentra en juego es la posibilidad de tomar la vanguardia en el enfrentamiento y poder acumular en el conflicto. Si el poder es omnipresente, las organizaciones populares pierden la posibilidad del "efecto sorpresa" en las acciones directas. El cuerpo se encuentra acorralado e invadido, el sentimiento de pérdida de la privacidad resulta frecuente: denuncian tener los celulares intervenidos, control en las redes sociales y, en otros casos, se aplica el seguimiento personalizado a los miembros de los colectivos en lucha. El estado de excepción asume una crudeza y virulencia que deja profundas marcas en la subjetividad. En los relatos existe un antes y un después que da cuenta del temor que siente el cuerpo que ha sido sometido al control y la violencia.

Así, en el discurso se refuerza la idea de un sistema político "corrupto" que funciona con base en un mecanismo de arbitrariedad selectiva que, en este caso, se entrelaza con la aplicación de la ley, acompañada por el uso de la fuerza y el amedrentamiento. Las entrevistadas lo expresan así: 
Claro, al principio era nuestro barrio y con el tiempo se fue sumando y, a ver, llegamos a ser, en un momento muy muy crucial debemos haber llegado a reuniones de más de 50 personas, hasta que José Porta- dueño de la empresa- nos sacó el Quincho del Tala, él pedía mucho una reunión, antes no quería hablar con nosotros y después [...], pero como no salió conforme de la reunión, pudo ir ahí con su hijo y nadie lo agredió. Lo dejamos hablar y después le dijimos las verdades, no le gustó, entonces a la semana mandó un patovica municipal a decirle a un técnico, un inspector, a decirle al Quincho del Tala que tenía que cerrar, que no tenía todas las condiciones, que tenía que cambiar el techo; ellos tenían matafuegos, hasta el momento tenían habilitación pero se ve que para determinada categoría tenía que tener otro techo. Pero es un salón precioso que tenía ingreso de seguridad que tenía salida por otro acceso, tenía techo en todo el salón. ¡Es un salón! No, le quieren hacer quitar ese techo, poner otro techo. La cuestión es que cuando te quieren joder la vida te la joden, yo te puedo asegurar, ellos no tenían habilitación de bomberos y si vos te ponés un kioskito y no tenés matafuego te lo van a clausurar (vUDAs, 54: 26).

Así, se construye un imaginario de un sistema político configurado para defender los intereses de los sectores adinerados y empresariales en detrimento de los sectores trabajadores. En ninguna de las citas analizadas se expresa la idea de un "mal funcionamiento" del sistema político. Por el contrario, esta emerge como la característica principal del sistema político en general.

En este sentido, aparecen palabras como "patovica” para nominar a un técnico de habilitaciones edilicias. Este término refiere al empleo en seguridad privada desempeńado por personas por lo general muy fornidas, que administran el ingreso a locales de uso social, de acuerdo con las características físicas de la persona que desee entrar. De esta manera, el uso de la palabra "patovica" para designar a un funcionario público refuerza la arbitrariedad en las decisiones y su apoyo en la fuerza y coerción.

En la siguiente cita se expresa una nueva expresión del mecanismo de arbitrariedad selectiva a partir del miedo que se produce ante la posibilidad de pérdida de los bienes materiales en el marco del conflicto:

Es muy difícil, es muy difícil. A mí me costó conquistar a mi familia, Porta nos clausuró este lugar; por ejemplo, después de que hicimos las asambleas nos llegó la municipalidad y no eran inspectores comunes, eran altos funcionarios de la municipalidad que venían a clausurar este lugar de mierda, tenían un interés. 
El sistema político como dispositivo de poder en un conflicto medio ambiental en Argentina

Entonces mi vieja se retiró de la lucha, tuvo miedo, la gente tiene miedo, tiene miedo a perder su negocio, a perder su casa. Ahora para el otro juicio tenemos que responder con los bienes, si perdemos el juicio perdemos los bienes. Entonces en el primero, que no respondíamos con los bienes somos 19 vecinos (vUdAs, 55: 16).

En este conflicto se ejerce una coacción sobre los bienes materiales de los sujetos que participan en la lucha. Quienes se encuentran organizados sufren el riesgo de la posibilidad de perder sus bienes personales. Así, el mecanismo de la arbitrariedad selectiva se aplica sobre los cuerpos en lucha y, también, sobre sus bienes materiales. La represión ataca la integridad del cuerpo y también sus condiciones de posibilidad, el lugar de refugio y aquello que genera los ingresos que hacen posible la vida. De esta manera, emerge el miedo como resultado del funcionamiento del estado de excepción. Este temor solo puede ser desarticulado a partir de la "conquista" de los individuos, es decir, de lograr el convencimiento.

En síntesis, en este apartado hemos analizado el funcionamiento del dispositivo del poder político que se desprende de los discursos analizados. Este dispositivo articula diversos mecanismos de poder: oscuridad del sistema, control de los flujos de información y arbitrariedad selectiva. Todos ellos se despliegan para controlar el conflicto y lograr su distensión. El control de los cuerpos y territorios se expresa como omnipresente en tanto la vida en su totalidad se encuentra atravesada por la vigilancia. La posibilidad de crecimiento de la lucha se encuentra contenida frente a los bloqueos que producen los mecanismos de poder. Así, los cuerpos y el territorio se encuentran sumidos en un estado de excepción donde todos los derechos son vulnerados, por lo cual los cuerpos deben esforzarse en la creación de prácticas de protección y nuevas formas de lucha.

\section{Estrategias: la exposición selectiva de los cuerpos}

El estado de excepción que se configura en el conflicto analizado expone el cuerpo a un alto nivel de control y violencia para distender el conflicto y desarticular la producción territorial. Sin embargo, a pesar de ello, los sujetos elaboran estrategias que permiten la protección y reducción de la exposición. Así, emerge la exposición selectiva de los cuerpos. Frente a situaciones de gran violencia y control, existe una decisión colectiva sobre qué cuerpos deben exponerse en contextos de lucha: 
ER1: O sea que eso vos decís que es como el contexto que dificulta la acción directa. EO1: Totalmente, en realidad... el primer camión me senté con Emilia, que es la que está allá de 7 añitos, el primer camión lo detuvimos Emilia y yo, pensá, Emi y yo nos sentamos en la calle y fue el primer camión ese día. Si hubieran estado los medios nos garantiza algún tipo de protección. ER1: Pero no están. EO1: No, no están, no están y ahí, bueno, era ella chiquita, por eso me imagino que no pasó nada después, bueno, ya directamente nos levantaban y nos sacaban, ni un medio. ER1: ¿No vino ni uno? EO1: Vino Canal 8, que nunca había venido y estuvo un ratito, en el momento en que se puso más duro; cuando decidieron "pasamos o pasamos y los levantamos a los vecinos" yo estaba trabajando, pero mi hija estuvo ahí y la pasó bastante mal y no hubo ningún medio. Entonces eso hace que las acciones directas... es un modo de, poner mucho el cuerpo, corres riesgo, corre riesgo tu vida, la de tus hijos, los vecinos y que no haya ningún medio es a propósito (vUDAS, 55: 13).

En esta cita, la entrevistada relata una acción directa que consistió en un bloqueo frente al ingreso de la planta Porta Hermanos. El bloqueo se llevó adelante al impedir el acceso o la salida de la producción mediante una sentada frente al portón principal.

Este extracto da cuenta de la desprotección y el desamparo total que experimentan los miembros de los colectivos en lucha. La ausencia de los medios de comunicación en las acciones directas es significada con el "a propósito" y produce condiciones de exposición y precariedad para quienes se encuentran en la lucha. En estos contextos, "poner el cuerpo" aparece como la única opción. De esta manera, el cuerpo asume una centralidad clave, ya que es aquello que está en juego. La exposición selectiva de algunos cuerpos ${ }^{8}$ produce las condiciones de protección para todo el colectivo en lucha. En particular, la participación de niños y niñas en acciones directas demarca límites al accionar del estado de excep-

8 En los otros casos analizados, que no son expuestos aquí por razones de extensión, emerge esta misma estrategia de exposición selectiva de los cuerpos como un modo de producir protecciones colectivas. En estos casos, la exposición selectiva se vincula a mujeres que asumen funciones de gran exposición y visibilidad política en contextos de represión y ausencia de garantías. De esta manera, a través del uso estratégico de la construcción social de la desigualdad de género, estas mujeres se configuran y afirman como sujetos políticos en lucha y resignifican la marca que sobre ellas se ha erigido. La visibilidad de las mujeres en estas luchas no solo es relevante desde el punto de vista político, sino que, a su vez, exige la puesta en marcha de mecanismos de poder particulares, conforme lo señala la ley. Si bien se trata de estados de excepción, que trascienden y rompen con lo regulado, la exposición selectiva de mujeres aspira, en los casos estudiados, a producir marcos de garantías y protección colectiva. 
El sistema político como dispositivo de poder en un conflicto medio ambiental en Argentina

ción. Así, cuando el sistema político ha bloqueado todas las estrategias posibles, el cuerpo es lo que queda.

\section{Reflexiones finales}

En este apartado hemos analizado el funcionamiento del dispositivo del poder político que se desprende de los discursos analizados. No se trata de la totalidad del sistema político, en general, sino de la particularidad que asume en los conflictos para su distensión y administración. Este dispositivo articula los partidos políticos, la justicia, los medios de comunicación y los representantes del Gobierno en todos los niveles, desde el más local y barrial hasta los niveles provinciales.

En los discursos analizados emerge un ideario de un juego de posiciones conformado por un "nosotros" -el colectivo en lucha- y un "ellos" -la empresa-, en articulación con el dispositivo político. Así, el dispositivo político se configura y asume sentido para hacer posible las necesidades de la empresa Porta Hermanos y pone en funcionamiento diversos mecanismos de poder para la reproducción del capital.

Un primer mecanismo de poder es la "oscuridad" del sistema político. Esta analogía refiere al ideario de un sistema político difuso y poco claro que asume esta condición cuando se despliega sobre procesos conflictivos. Así, la oscuridad no es entendida como una disfunción del sistema, sino como un mecanismo de poder cuya función es dificultar su inteligibilidad y difuminar las responsabilidades de las decisiones represivas. En el discurso de los miembros de vuDas emerge constantemente una desconfianza frente a los mecanismos legales y un ideario de lucha a "ciegas" que refiere a la imposibilidad de dimensionar frente a qué o a quiénes se enfrentan y quiénes toman las decisiones que deben enfrentar.

El segundo mecanismo de poder es el control de los flujos de información. Refiere a la vigilancia que se establece sobre los movimientos y la información de los miembros del colectivo en lucha. De este modo, las acciones directas planificadas por el colectivo pierden efectividad, ya que la filtración de la información dificulta su efecto sorpresa. En otras palabras, se pierde la posibilidad de generar un "avance" en el marco del juego de posiciones percibido. Frente a esto, las y los vecinos establecen la estrategia de reducir los círculos de circulación de la información y los canales comunicativos. 
El tercer mecanismo de poder es la arbitrariedad selectiva, entendiendo por esta la aplicación selectiva de la ley para desarticular el proceso organizativo. De este modo, la articulación de los tres mecanismos de poder en el marco del dispositivo del sistema político produce un sistema de bloqueos que impide el crecimiento del proceso de lucha. Los sentimientos de soledad y falta de alianzas políticas son constantes en estos discursos y refieren a un "encorsetamiento" del proceso conflictivo. El sistema político es percibido como omnipresente y se reducen las posibilidades de generar escenarios de disputa. En este sentido, emerge la estrategia de romper el cerco construido por la empresa y el dispositivo del sistema político a partir de trasladar el epicentro del conflicto a espacios de mayor visibilidad política, como la sede del municipio.

Todo lo aquí referido remite a la configuración de estados de excepción. Así, la oscuridad, el control de los flujos de información, la arbitrariedad selectiva y la omnipresencia del control remiten a "excepciones" en el ejercicio político y en los derechos de los sujetos. De allí que el estado de excepción se configure como un complejo y opaco entramado de relaciones de poder que se despliegan para controlar el conflicto. El sistema político funciona de acuerdo con los intereses del capital, lo que da lugar al ejercicio de estados de excepción. No existe "un" estado de excepción, sino "estados de excepción", es decir, parcelas de poder que, legal o ilegalmente, escapan de los límites establecidos por el Estado de derecho.

Los estados de excepción suponen suspensiones de los derechos y las garantías de los sujetos para dar lugar a acciones sobre los sujetos y territorios que están por fuera de la norma y que suponen el ejercicio del poder arbitrario. Sin embargo, no se trata de acciones que se opongan al ejercicio del Estado de derecho y que vayan a ser juzgadas o reguladas por el mismo, sino que, por el contrario, se trata de la estrecha vinculación que se construye entre Estado de derecho y excepciones que habilita la administración de la conflictividad social en favor de los sectores poderosos.

Frente al ejercicio del poder en el marco de los estados de excepción, los cuerpos deben esforzarse en la creación de prácticas de protección y nuevas formas de lucha. Este es el caso de la exposición selectiva de los cuerpos que busca generar condiciones de protección para la totalidad del proceso organizativo en contextos de estados de excepción. 
El sistema político como dispositivo de poder en un conflicto medio ambiental en Argentina

\section{Referencias}

Agamben, G. (2004). El estado de excepción. Archipiélago: Cuadernos de Crítica de la Cultura, (60), pp. 99-109.

(2010). Homo Sacer. El poder soberano y la nuda vida. Valencia: Pre-textos.

(2011). ¿Qué es un dispositivo? Sociológica, 26(73), pp. 249-264.

Abadía, O. M. (2003). ¿Qué es un dispositivo? Empiria. Revista de metodología de ciencias sociales, (6), pp. 29-46.

Avalle, G. (2014). La contienda de la educación: lucha y acción colectiva sindical en la Argentina contemporánea, análisis de tres contiendas provinciales de sindicalismo docente. Tesis doctoral sin publicar. Córdoba: Universidad Católica de Córdoba.

Ciuffolini, M. A. (2010). Resistencias: Luchas sociales urbanas en Córdoba post-2001. Córdoba: EDUCC.

De Certeau, M. (1996). La invención de lo cotidiano. México: Universidad Iberoamericana.

Diario Cba 24n (2013, 13 de agosto). Acusan que el 50\% del barrio está enfermo por planta de Porta. Cba 24n. Recuperado de https://www.cba24n.com.ar/content/acusan-que-el-50-del-barrio-esta-enfermo-por-planta-de-porta.

Diario La voz (2014, 8 de noviembre). Aún hay 200 industrias fuera de norma. La voz. Recuperado de https://www.lavoz.com.ar/ciudadanos/aun-hay-200-industrias-fuera-de-norma.

Foucault, M. (1988). El sujeto y el poder. Revista mexicana de sociología, 50(3), pp. 3-20. (1994). Dichos y escritos. Tomo III. Madrid: Editorial Nacional.

Guattari, F. \& Rolnik, S. (2008). Micropolítica. Cartografías del deseo. Madrid: Traficantes de Sueños.

Muñiz, M. (2010). Estudios de caso en la investigación cualitativa. División de Estudios de Posgrado Universidad Autónoma de Nuevo León. Facultad de Psicología, pp. 1-8.

Murillo, S. (2008). El conflicto social en Michel Foucault. Revista Conflicto Social, 1(0), pp. 156-180.

Neiman, G. \& Quaranta, G. (2006). Los estudios de caso en la investigación sociológica. Estrategias de investigación cualitativa, (1), pp. 213-237.

O'Connor, J. (2000) ¿Es posible el capitalismo sostenible? Papeles de población, 6(24), pp. 9-35.

Svampa, M. (2013). Consenso de los Commodities y lenguajes de valoración en América Latina. Nueva sociedad, (244), pp. 30-46.

Valim, R. (2018). Estado de excepción: la forma jurídica del neoliberalismo. Revista Derechos en Acción, (7), pp. 438- 461.

Vasilachis de Giardino, I. (2005). La representación discursiva de los conflictos sociales en la prensa escrita. Estudios sociológicos, pp. 95-137.

Zibechi, R. (2006). Movimientos sociales: nuevos escenarios y desafíos inéditos. OSAL, Observatorio Social Latinoamericano, (21), pp. 221-230. 